\title{
Molecular Background of miRNA Role in Asthma and COPD: An Updated Insight
}

\author{
Izabela Szymczak, Joanna Wieczfinska, and Rafal Pawliczak \\ Department of Immunopathology, Faculty of Medical Science and Postgraduate Training, Medical University of Lodz, \\ Zeligowskiego 7/9 Street, Building 2, Room 122, 90-752 Lodz, Poland \\ Correspondence should be addressed to Rafal Pawliczak; rafal.pawliczak@csk.umed.lodz.pl
}

Received 16 February 2016; Revised 29 April 2016; Accepted 15 May 2016

Academic Editor: Andras Bikov

Copyright (C) 2016 Izabela Szymczak et al. This is an open access article distributed under the Creative Commons Attribution License, which permits unrestricted use, distribution, and reproduction in any medium, provided the original work is properly cited.

\begin{abstract}
Inflammatory airway diseases are a significant health problems requiring new approaches to the existing therapies and addressing fundamental issues. Difficulties in developing effective therapeutic strategies might be caused by lack of understanding of their exact molecular mechanism. MicroRNAs (miRNAs) are a class of regulators that already revolutionized the view of gene expression regulation. A cumulating number of investigations show a pivotal role of miRNAs in the pathogenesis of asthma, chronic obstructive pulmonary disease (COPD), or airway remodeling through the regulation of many pathways involved in their pathogenesis. Expression changes of several miRNAs have also been found to play a role in the development and/or improvement in asthma or COPD. Still, relatively little is known about the role of miRNAs in inflammatory disorders. The microRNA profiles may differ depending on the cell type or antigen-presenting cell. Based on the newest literature, this review discusses the current knowledge concerning miRNA contribution and influence on lung inflammation and chosen inflammatory airway diseases: asthma and COPD.
\end{abstract}

\section{Introduction}

Noncoding RNAs, housekeeping RNAs, long noncoding RNAs, and small noncoding RNAs (endogenous short interfering RNA and microRNAs (miRNAs)), have emerged as important molecules in lung disease [1], out of which, in lung diseases, the miRNAs are most commonly investigated. miRNAs are short, endogenous, single-stranded, noncoding, and consisting of 18-25 nucleotides RNAs and evolutionary conserved sequences that regulate both expression of genes and gene networks [2]. They are capable of inducing degradation of messenger RNA (mRNA) and/or repression of mRNA translation. Targets for miRNAs may be approximately $60 \%$ of mRNAs [3]. miRNAs have a role in controling the signaling pathways in many types of cells and in phenotype and development of cells of the immune system as well as regulation of the inflammatory response in many tissues [4].

The biogenesis of miRNA begins in the nucleus. The primary miRNAs are transcribed by RNA polymerase II [2] and cleaved by Drosha, a nuclear RNAse III enzyme [5-7]. The resulting pre-miRNA is transported into the cytoplasm and processed into double-stranded miRNA by Dicer, a RNAse III enzyme. One strand of this duplex represents the mature miRNA and is assembled into the RNA-induced silencing complex (RISC), while the other strand of this duplex is degraded. miRNA incorporated into the RISC regulates expression of genes by translational repression or degradation of mRNA, which depends on complementarity of sequence between mRNA $3^{\prime}$ untranslated region and miRNA $5^{\prime}$ region $[2,8,9]$. This review focuses on relationship between miRNAs and inflammatory pulmonary diseases. Additionally, the association of miRNAs with molecular pathways involved in inflammation coexisting with pulmonary diseases was summarized.

\section{Inflammation}

Inflammation is a process aiming at neutralizing or removing harmful factors such as damaged cells, allergens, irritants, pathogens, toxins, and initiating healing process in the tissue. In healthy individuals, the immune system is very efficient 
in detecting even a very small number of pathogens as well as eliminating them, before they have reached harmful numbers. However, inadequate or excessive responses may lead to severe inflammation as well as tissue damage [10-12].

It has been demonstrated that several miRNAs play an important role in the regulation of different inflammatory processes related to asthma, COPD (chronic obstructive pulmonary disease) and airway remodeling [13-15]. The need for research on microRNA molecules is confirmed by the fact that many studies have been registered as clinical trials. Over 20 clinical trials, evaluating miRNA in inflammation, are registered, 6 of which are concerned only about airway diseases, whereas 6 and 8 trials tackle problems of asthma and obstructive lung disease, respectively (https://clinicaltrials.gov/).

\section{Asthma}

Asthma is a respiratory disease, characterized by limitation of air flow and chronic inflammation due to persistent infiltration of mast cells and eosinophils, related to poor Th2 response or not associated with this immune process [16]. Environmental and genetic factors play a role in the pathogenesis of the disease. IL-4, IL-5, IL-9, and IL-13 are Th2 cytokines involved in hypersensitivity of airway, elevated secretion of mucus, and increased IgE levels and infiltration of eosinophils [17].

Recently, the number of studies on miRNA role in asthma has elevated, indicating various expressions of miRNA in different models of asthma. Despite the fact that the methods of experimental induction of asthma are different, the same phenotypes are demonstrated by them, like Th2-related inflammation, eosinophil airway recruitment, elevated airway hypersensitivity, and secretion of mucus. Different expression of 21 miRNAs in allergic asthma and healthy individuals has been shown in mouse models of allergic asthma [18]. Some studies demonstrated that the expression of miR-21 increased in asthma models and was related to Th2 response and IL-12 expression [18, 19]. It has been observed in Aspergillus fumigatus, ovalbumin, house dust mite, and lung specific IL-13 models of asthma [18].

Lower levels of eosinophilia, decreased IL-4, and elevated INF- $\gamma$ in bronchoalveolar lavage fluid were observed in ovalbumin-induced model of asthma in mice with lack of miR-21, after allergens' exposure, compared to the controls [19]. Additionally, lack of miR-21 in CD4+ T cells resulted in decreased IL-4 and increased INF- $\gamma$ levels [19].

Various asthma models were documented to have different expression of miRNAs. Wang et al. identified different expression of miR-145-5p, miR-636, miR-338-3p, miR-4485, miR-1229-3p, miR-4707-3p, and miR-3620-3p in the serum of patients with asthma, compared to patients with COPD [20]. A few studies demonstrated different expression of 11 miRNAs in the exhaled breath condensate (EBC) from patients with asthma compared to healthy individuals [21]. Roff et al. also detected decreased levels of miR-570-3p in $\mathrm{EBC}$ and in the serum from the patients with asthma. Inverse correlation between levels of miR-570-3p and lung function was documented, based on the observation of diverse effect on chemokine and cytokine expression in human epithelial cells of airways with upregulation of IL-6, tumor necrosis factor (TNF), chemokine (C-C motif) ligand 4 (CCL4), CCL5 in response to TNF, suppression of TNF-induced chemokine (C-X-C motif) 8, and CCL2 [22]. Significant differences in exosomal miRNA from bronchoalveolar lavage fluid was observed in mild asymptomatic asthma patients in comparison with healthy individuals [23]. Figure 1 shows the role of miRNAs in asthma.

Expression of miR-126 and miR-21, identified in epithelial cells of airway in patients on inhaled corticosteroids, was decreased whereas it was upregulated in patients with asthma. Thus, the miRNAs may be related to asthma and may be a biomarker of the therapy [32].

Different expression of miRNAs, mRNAs, and ncRNAs was detected in human primary airway smooth muscle (ASM) cells following corticosteroid treatment and mitogen stimulation [28]. Altered miR-221 expression was documented in ASM cells from patients with nonsevere asthma and those with severe asthma and healthy individuals. miR221 also regulates inflammatory state and aberrant proliferation observed in ASM cells [26]. The regulatory role of miR146a in inflammation in ASM cells from patients with asthma was confirmed. Decreased RNA binding protein human antigen $\mathrm{R}(\mathrm{HuR})$ expression is related to downregulation of cyclooxygenase-2 (COX-2) via miR-146a [33]. Proinflammatory cytokines induced expression of miR-146a and miR$146 \mathrm{~b}$ in ASM cells; expression of miR-146a was higher in cells derived from patients with asthma. miR-146a may be determined as a negative endogenous regulator in ASM cells. Thus, the potential candidate used in anti-inflammatory asthma treatment may be miR-146 mimics. The direct miR570-3p target is also HuR. Comer et al. reported that miR155 is involved in $\beta 2$-adrenoceptor hyperresponsiveness and regulates COX-2 and secretion of prostaglandin E2 from ASM cells of humans [34]. Positive correlation of miR-155 and COX-2 expression in ASM cells from asthmatic patients was observed. miR-10a is a regulator of mitogen-induced proliferation of ASM via targeting the phosphoinositide 3kinase (PI3K) pathway by inhibition of the subunits of PI2K and PIK3CA expression [35].

Exacerbation of asthma may be caused by many gaseous components of environmental pollutants, that is, ozone. Ambient particulate matter and particles of diesel exhaust may upregulate thymic stromal lymphopoietin (TSLP), which is cytokine linking Th2 adaptive immune and innate disorders, as well as miR-375 expression in human epithelial cells of bronchi [27]. Upregulated TSLP in human epithelial cells of bronchi is associated with interaction between miR375 and regulatory effects upon the aryl hydrocarbon mRNA. Ozone is related to many adverse effects on health and elevated levels of miRNAs including miR-199a, miR-199bp5, miR-132, miR-424, miR-223, miR-143, miR-222, miR-582$5 \mathrm{p}, \mathrm{miR}-25$, and miR-145 expression in bronchial airways of humans [36]. Environmental dust pollution may cause dyspnea, linked with alterations in expression of miRNA in bronchoalveolar lavage fluid, urine, and serum, found in military personnel serving in Afghanistan and Iraq. Interestingly, different overexpression of miR-371-5p was observed in these fluids [37]. 


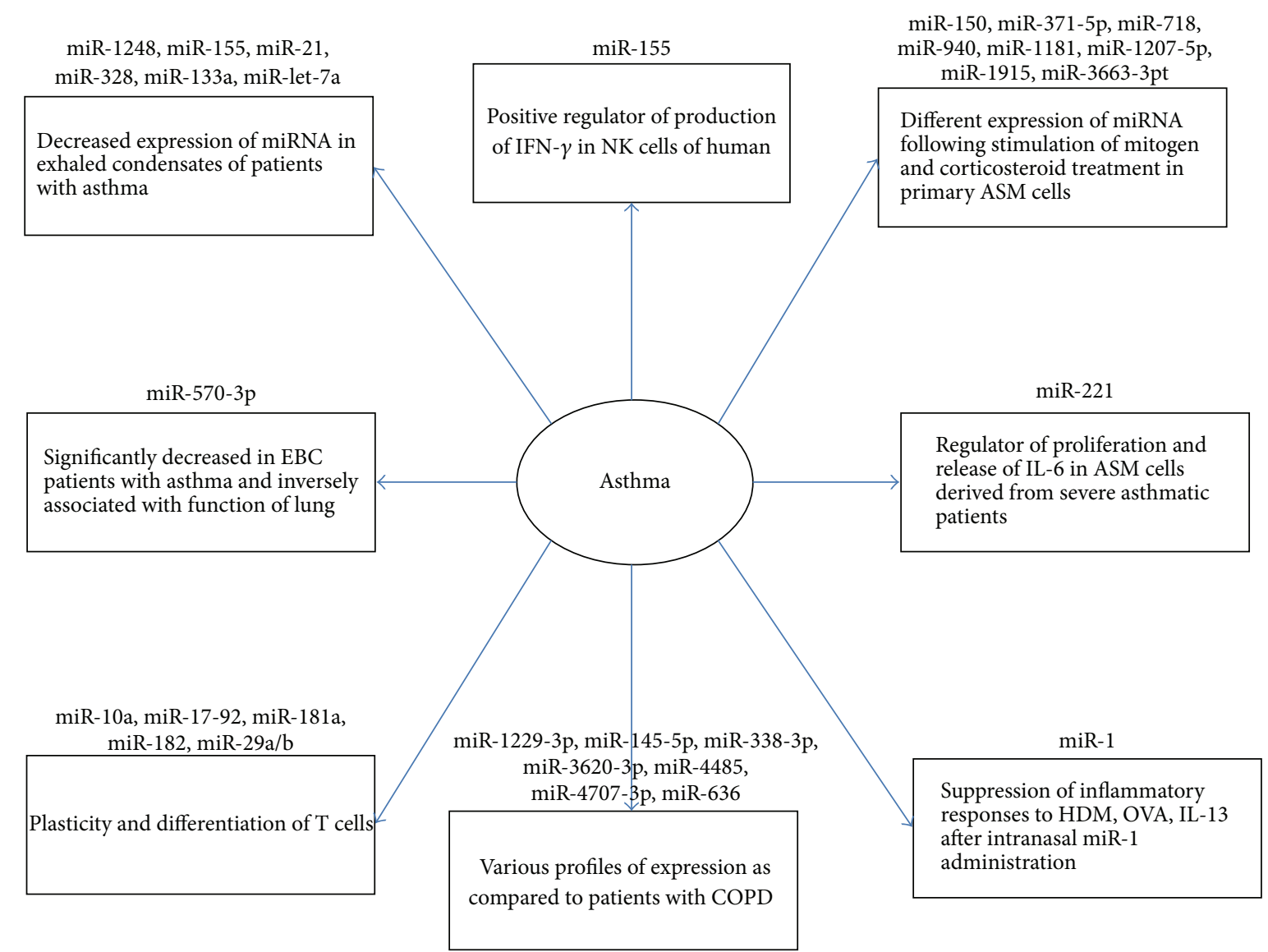

FIGURE 1: The role of miRNAs in asthma [20, 22, 24-30], in recent studies.

Not only expression of miRNA but also genetic background may be related to response to the drug treatment. The miR-148a, miR-148b, and miR-152 inhibited expression of major histocompatibility complex, classes I and G, and single nucleotide polymorphism in the $3^{\prime}$-UTR region of HLA-G modulated binding of miRNA. Individuals with the $\mathrm{G}$ minor allele of the single nucleotide polymorphism showed reduced exacerbation of asthma in comparison with the individuals who did not carry the $G$ minor allele after treatment with statins [38].

\section{COPD}

COPD and asthma are diseases that cause chronic inflammation of the airways but have distinct characteristics. COPD is characterized by the presence of chronic obstruction and is not very reversible to airflow associated with an anomalous inflammatory reaction, mainly tobacco smoke. In COPD the cellular composition of the airway inflammatory infiltrate contains mainly neutrophils, macrophages, and lymphocytes. In contrast, asthma is partially conditioned by genetic factors and runs its course with bronchial hyperresponsiveness and airflow obstruction, which either totally or partially reverses spontaneously or by medicinal action. Eosinophils are the most prominent inflammatory cells in asthma, with mast cells, lymphocytes, and macrophages playing important but less prominent roles. The contrasting inflammatory phenotypes of asthma and COPD have important implications for clinical and physiologic manifestations of disease, as well as for therapy $[39,40]$.

Similarly to asthma, a pathophysiological role for miRNAs in COPD has been suggested in several studies, in which differential expression of miRNAs has been identified in lung cell (Figure 2) [41, 42].

Cao et al. detected increased expression of miR-183, miR$200 \mathrm{~b}$, and miR-200c in the peripheral blood in patients with COPD but found no significant difference between different severities of COPD. Their study suggests that increased expression of miR-183 in COPD patients' peripheral blood might be involved in regulation of $\mathrm{BKCa} \beta 1$ expression, which in turn might be correlated with the severity of the disease. $\mathrm{BKCa} \beta 1$ and miR-183 could be therefore considered promising biomarkers for clinical diagnosis and treatment of COPD [43].

Furthermore, Donaldson et al. found that, in patients with stable COPD, plasma levels of muscle-specific miRNAs are increased, suggesting that muscle wasting or turnover is increased even in samples from patients with stable COPD [44]. The study showed that plasma levels of musclespecific miR-499 are associated with nuclear NF- $\kappa$ B p50 in 


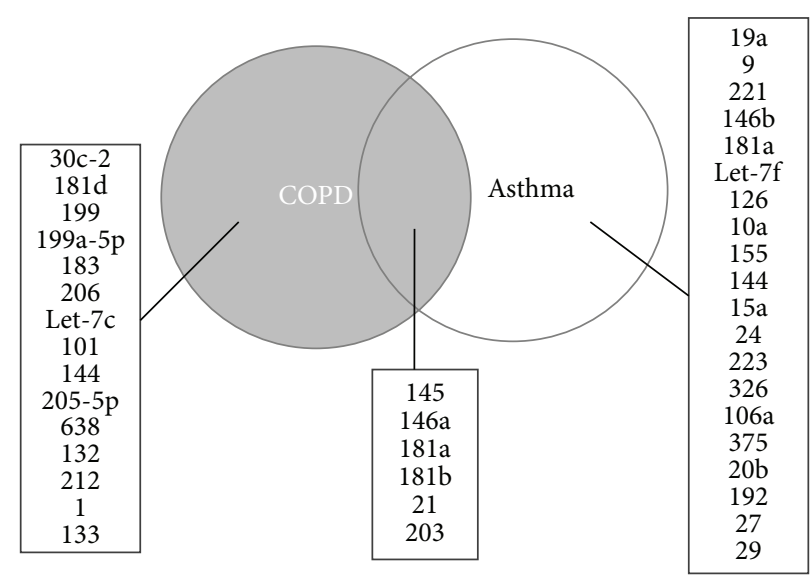

FIGURE 2: Venn diagram of microRNAs (miRNAs) associated with chronic obstructive pulmonary disease (COPD) and/or asthma. The lists show the miRNAs associated with each component of the diagram, based on Molina-Pinelo et al. [31].

mild/moderate COPD, whereas, in severe and very severe disease, miR-206 and miR-133 are associated with circulating cytokines. This observation raises the possibility that inflammation is an important driver of wasting in this patient subgroup.

The expression of numerous inflammatory mediators associated with COPD pathogenesis is controled by NF- $\kappa \mathrm{B}$. In $\mathrm{COPD}$, higher levels of activated $\mathrm{NF}-\kappa \mathrm{B}$ are observed in the bronchial biopsies and inflammatory cells of individuals [45]. Moreover, plasma levels of muscle-specific miR-499, miR133, and miR-206 were elevated in COPD patients compared to the healthy controls, but, among the patients, the highest plasma levels were associated with better lung function [44]. This paradox was explained by differences in the muscle pool in patients with mild versus severe disease to release miRNA. The decrease in miR-499 observed in this study in patients with severe lung function impairment is consistent with observations from patients with lung cancer in whom low plasma levels of miR-499 were a predictor of reduced survival, perhaps as a result of cachexia [46]. As plasma miR499 levels are directly correlated with nuclear NF- $\kappa$ B p50 in COPD, this might suggest that loss of type I fibres or export of miR-499 is dependent on NF- $\kappa$ B activation [44].

Aside from the classic transcription factors, musclespecific miRNAs play a relevant role in the regulation of muscle development and repair after injury by targeting different pathways. In COPD, the inspiratory loads to which the respiratory muscle is continuously exposed may be a major player accounting for specific pattern of miRNA expression [47]. In the main inspiratory muscle (diaphragm) of patients with mild-to-moderate and severe COPD, expression of some muscle-specific miRNAs miR-1, miR-133, and miR-206 is downregulated. However, the expression of miR-486, miR27a, miR-29b, and miR-181a does not differ between the patients and the controls [47].

Muscle-specific miRNAs were analyzed also by PuigVilanova et al. [47]. As the miRNAs (miR-1, miR-133, and miR-206) were downregulated, it is likely that epigenetic events act as biological adaptive mechanisms to better overcome the continuous inspiratory loads of the respiratory system in COPD. Histone deacetylase 2 level was upregulated in the respiratory muscle of COPD patients in this study. Another study presented data from lung tissues of COPD patients, displaying that the reduction in lung tissue histone deacetylase 2 expression may be related to the regulation of both $\mathrm{p} 53$ and hypoxia-inducible factor- $1 \alpha$ (HIF- $1 \alpha)[47,48]$.

It has been reported that the expression of HIF- $1 \alpha$ is reduced and p53 is increased in lungs from patients with COPD in comparison with the healthy controls. Mizuno et al. found overexpression of miR-199a-5p and miR-34a in the lung tissues from patients with COPD, compared to normal lungs. Moreover, increased expression of miR-34a was induced by upregulation of $\mathrm{p} 53$, and HIF- $1 \alpha$ protein expression was inversely correlated with the expression of miR-199a-5p. The expression of miR-34a and miR-199a-5p was related to lung disease severity; the authors found also correlation between the expression of miR-34a and miR199a-5p [49]. These data might suggest that miR-34a and miR-199a-5p not only contribute to COPD pathogenesis but also may affect HIF-1 $\alpha$-dependent lung structure maintenance program.

Distinct miRNA expression profiles were identified and validated in the study published by Molina-Pinelo et al. for each pathological group, involving 66 differentially expressed miRNAs [31]. miR-132 and miR-212 were upregulated in patients with COPD; however, no apparent correlation was observed between the burden of cigarette smoking and expression patterns of the miRNAs. Moreover, the authors also found a negative correlation between $\alpha 1$-antitrypsin mRNA and miR-132-212 cluster expression. Consistent with these findings, $\alpha$-antitrypsin mRNA has been recently described as a target for the miR-132-212 cluster, and its deficiency has been involved in COPD development [50, 51].

Diseases due to cigarette smoke exposure, including COPD, are associated with chronic inflammation typified by the increased expression of COX-2 protein. RelB is a protein that suppresses cigarette smoke induction of COX2 through an unknown mechanism [52]. Zago et al. showed that endogenous expression of miR-146a in murine lung fibroblasts is dependent on RelB expression (which plays 
a role in reducing cigarette smoke-induced COX-2 protein expression). Moreover, suppression of cigarette smokeinduced COX-2 protein occurs via miR-146a, so possibly a functional association exists between RelB and miR-146a in the suppression of COX-2 protein by cigarette smoke exposure [52]. Cigarette smoke contributes to COPD by inciting inflammation, recruiting T cells, macrophages, and neutrophils to the lung, in part via the induction of inflammatory mediators, including COX-2; therefore miRNA participation in this process might be a promising issue.

COPD is a heterogeneous disease, and it is possible that some miRNAs may be differently expressed only in a subset of patients with COPD. From a clinical perspective, the targeting of noncoding RNAs as a novel therapeutic approach requires a deeper understanding of their function and mechanism of action. However, in the short term, changes in miRNA are likely to be of use as biomarkers for disease stratification and/or assessment of drug action $[1,39]$.

\section{Airway Remodeling}

ASM cells and bronchial epithelium are considered a key player in coordinating airway wall remodeling. Current therapeutics for asthma are effective in reducing inflammation; however, many therapies even long term pharmacological therapies do not reprogram the underlying immune deviation that drives the pathology. For this reason, there is a crucial need for developing new strategies to decrease or reverse the ongoing remodeling process $[53,54]$. Airway remodeling is amplified by profibrotic mediators, such as transforming growth factor- $\beta$ (TGF- $\beta$ ), which plays a cardinal role in various models of fibrosis [55].

Haj-Salem et al. proved that proliferation rate of epithelial cells of severe asthmatic patients is higher than in controls and mild asthmatics and that miR-19a increases proliferation in severe asthma through the downregulation of TGF- $\beta$ receptor 2 [56]. They demonstrated that miR-19a itself is specifically increased in airway epithelial cells of severe asthmatics in comparison with mild asthmatic and healthy subjects. This is significant, as miR-19a belongs to the cluster miR-17 92 that plays a key role in the control of cell cycle and cell death. Moreover, repressed expression of miR-19a increased Smad3 phosphorylation through TGF- $\beta$ receptor 2 signaling and abrogated bronchial epithelial cell proliferation [56].

Transforming growth factor $\beta 1$ (TGF- $\beta 1$ ) is an important mediator participating in the lung fibrosis development in patients with severe asthma $[57,58]$. In vitro, it may also induce migration as well as proliferation of smooth muscle cells $[59,60]$. Because of the key effects of TGF- $\beta 1$ in these processes, it may be used as a model of remodeling and inflammation [61].

The effect of TGF- $\beta$ is mediated by its interaction with transmembrane receptors. Three types of receptors can be distinguished: type I, TGF $\beta$ R1, type II, TGF $\beta$ R2, and type III, TGF $\beta$ R3. Only autophosphorylated TGF $\beta$ R2 may bind TGF- $\beta$. After binding with TGF- $\beta$, TGF $\beta$ R1 is recruited and phosphorylated by TGF $\beta$ R2. Then, TGF $\beta$ R1 initiates transduction of signal that is mediated by Smad proteins [62]. The crucial role in the regulation of proliferation of airway smooth muscle cells and remodeling of airway is played by Smad3 proteins. Chen et al. demonstrated that the expression of miR-23b is inhibited by the miR-23b inhibitor sequence transfection and increased by the miR23b mimics sequence transfection in ASMCs. The level of TGF $\beta$ R2 expression is negatively regulated by miR-23b and miR-23b overexpression, suppressing remodeling of airway. Moreover, TGF- $\beta 1$-induced proliferation of airway smooth muscle cells is controled by miR-23b via TGF $\beta$ R $2 / \mathrm{p}-\mathrm{Smad} 3$ signals [63]. It has been reported that miR-23b prevents multiple autoimmune diseases through the regulation of inflammatory cytokine pathways, in which the molecule regulates a number of inflammatory cytokines, such as TGF$\beta 1, \mathrm{NF}-\kappa \mathrm{B}, \mathrm{TNF}, \mathrm{IL}-1$, and IL-17 [63].

There is an emerging evidence that miRNAs are important regulators of gene expression in the immune system, and this is supported by recent studies demonstrating a role for miRNAs in allergic airway inflammation $[18,64]$.

Allergen-induced inflammation is associated with remodeling responses in the airways of asthmatic patients, and the Th2 cytokines IL- 4 and IL-13 play an important role in this process. Malmhäll et al. reported that miR-155 is involved in the regulation of allergen-induced Th2-mediated eosinophilic inflammation in the airways [64]. miR-155 deficiency resulted in diminished eosinophilic inflammation and mucus hypersecretion in the lungs of allergen-sensitized and allergen-challenged mice compared to WT control animals. Furthermore, the transcription factor PU.1, a negative regulator of Th2 cytokine production, was upregulated in the airways of allergen-challenged miR-155 knockout mice compared to the controls. The authors also demonstrated that miR-155 is involved in the local regulation of Th2 responses in allergen-induced eosinophilic airway inflammation and highlighted the role of miR-155 as a potential target in patients with allergic inflammatory diseases [64].

Nonreceptor tyrosine kinase and Abelson tyrosine kinase (c-Abl) play an important role in regulating actin cytoskeleton that is important in many cellular functions such as migration and adhesion of cells [65-67]. Recently, it has been known that c-Abl plays a role in regulation of smooth muscle cell proliferation, which contributes to the development of airway remodeling in chronic asthma. Expression of c$\mathrm{Abl}$ is increased in asthmatic airway smooth muscle cells, and the factor regulating c-Abl expression is miR-203 [23]. Activated $\mathrm{c}-\mathrm{Abl}$ induces polymerization of actin regulating activation and recruitment of Raf- 1 . Then, activated Raf1 induces ERK1/2 and MEK1/2 enhancing proliferation of smooth muscle cells. However, c-Abl does not activate AKT that is also protein kinase implicating survival and growth of cells [68-71].

Based on the results of animal studies, it may be suggested that c-Abl may trigger pathogenesis of asthma [72, 73]. Expression of c-Abl is upregulated in cells of airway smooth muscle from asthmatic tissue of smooth muscle derived from animal model of asthma. c-Abl knockout suppresses allergeninduced airway remodeling in animal model of asthma [72]. Attenuation of airway thickening was also observed after 
treatment with inhibitor of c-Abl, imatinib in chronic animal model of asthma [74]. According to Levänen et al. miR-203 regulates smooth muscle cell proliferation by controling cAbl expression, which in turn modulates the activation of ERK1/2, which was confirmed by the fact that exposure to the miR-203 inhibitor increased the level of c-Abl in these cells. Moreover, the levels of miR-203 were shown to be diminished in asthmatic airway smooth muscle cells in this study [23]. Liao et al. demonstrated that miR-203 downregulates c-Abl expression, and PDGF-induced HASM cell proliferation, and reduces PDGF-induced ERK1/2 phosphorylation but does not affect phosphorylation of AKT in human airway smooth muscle cells. miR-203 expression is decreased in asthmatic human airway smooth muscle cells [75].

What is interesting, miR-203 has also been found overexpressed in the lung and blood samples of smokers. Shi et al. demonstrated that miR-203 functions as an immune response inhibitor through targeting TAK1 and PIK3CA [76]. Higher miR-203 expression and weaker NF- $\kappa$ B signaling activation were also detected in the COPD-diseased bronchial/tracheal epithelial cells, when stimulated by LPS. This particular micro-RNA deserves special attention as certainly it has a strong connection with airway diseases [76].

Recent study by Liu et al. displayed that miR-21 targeting may have detrimental effects on asthmatic airway remodeling [77]. According to the authors, miR-21 is significantly upregulated in smooth muscle cells of asthmatics, compared to nonasthmatic cells. The association of miR-2 with airway remodeling seems unquestionable, as the data indicate that miR-21 overexpression increased the proliferation and migration of ASM cells, which play a crucial role in asthma airway remodeling. Furthermore, miR-21 overexpression decreased the level of PTEN (phosphatase and tensin homolog) and activated the phosphoinositide 3-kinase (PI3K)/AKT pathway, while inhibition of miR-21 expression increased PTEN expression [77]. PTEN signaling pathway which is tightly associated with another targeted pathway, the PI3K/AKT pathway, leads to implementation of growth programs in cells from asthmatic patients. Moreover, the transcripts of six genes of this pathway belong to the list of 38 major targets for differently expressed miRNAs [78]. The phosphoinositide 3-kinase (PI3K) is protein catalyzing phosphorylation of phosphoinositides in $3-\mathrm{OH}$ position as well as controling various intracellular signaling pathways via generated lipids. Selective inhibitors of PI3K decrease inflammation and several characteristics of disease in animal models. It has been proposed that PI3K/AKT inhibitors may be a novel therapy in the treatment of COPD and asthma. $\mathrm{PI} 3 \mathrm{~K} / \mathrm{AKT}$ pathway is a cellular pathway involved in growth, differentiation, and survival of cells as well as expression of proteins and comprises also $3^{\prime}$-phosphoinositide dependent kinase-1 (PDK-1) [79].

The data published by the same team. Liu et el., revealed that another miRNA, miR-138, reduced proliferation of human airway smooth muscle cells. It also directly suppresses PDK-1 (3'-phosphoinositide dependent kinase-1) expression by targeting the $3^{\prime}$-UTR of the gene. miR-138 controls ASM proliferation through directly inhibiting the PI3K pathway [80]. Thus, $P D K-1$ gene has been identified as a direct target of miR-138 and is a key component in PI3K/AKT signaling, which is a pathway regulating ASM cell proliferation.

It has been suggested that numerous components of the PI3K pathway play crucial roles in the expression and activation of inflammatory mediators, inflammatory cell recruitment, immune cell function, airway remodeling, and corticosteroid insensitivity in chronic inflammatory respiratory diseases.

Aberrant inflammatory and remodeling responses in the lungs of asthmatic patients are thought to stem from deregulated gene expression, although the precise mechanism or mechanisms are still elusive [78]. Recent signaling pathway analysis demonstrated involvement of 38 miRNA-targeted mRNAs in increased cell proliferation through PTEN and PI3K/AKT signaling pathways [78]. Among them is the PPARG gene, known to be involved in airway inflammatory and remodeling responses, as well as adenosine $\mathrm{A} 2 \mathrm{~b}$ receptor (ADORA2B). Adenosine is a powerful bronchoconstrictor of asthmatic airways and not only acts as an inflammatory mediator in asthmatic patients but also participates in airway wall remodeling.

Relatively very few miRNAs have been studied in detail and, hence, the precise biological relevance remains yet to be determined. miRNAs have also been implicated to play a major role in airway disease regulation.

\section{6. $\mathrm{miR}-27 \mathrm{~b}$ and $1,25(\mathrm{OH})_{2} \mathrm{D}_{3}$}

Pulmonary fibroblasts play a crucial role in maintenance and formation of structure as well as function of lung and remodeling and repair of tissue that are crucial characteristics of chronic lung diseases, including pulmonary fibrosis, COPD, and asthma [81-86]. TGF- $\beta 1$ is produced by many types of cells and suggested to influence cell function, growth regulation, proliferation, apoptosis, differentiation, cell movement, and secretion and deposition of extracellular matrix. Moreover, TGF- $\beta 1$ regulates function and phenotype of fibroblasts [87-90].

Active metabolite of vitamin $\mathrm{D}, 1,25(\mathrm{OH})_{2} \mathrm{D}_{3}$, is able to suppress tissue remodeling response mediated by TGF- $\beta$ in fibroblast of lung and block TGF- $\beta 1$-mediated myofibroblastic transformation of fibroblasts. Some studies demonstrated association of TGF- $\beta$ and vitamin D signaling pathway. Vitamin D receptor (VDR) binds with Smad3 (MH1 domain) strengthening transactivation of Smad3 induced by ligand. Thus, active metabolite of vitamin $\mathrm{D}$ influences TGF- $\beta$ signaling pathway via alteration of Smad3 and VDR levels. VDR may be determined as a negative regulator of TGF- $\beta$-induced fibroblast differentiation. Expression of TGF- $\beta 1$-induced miR-27b is downregulated by $1,25(\mathrm{OH})_{2} \mathrm{D}_{3}$ in human fibroblast of lung. VDR expression in human fibroblast of lung is regulated via miR-27b that has capabilities of targeting VDR $3^{\prime}$-UTR and downregulation of VDR protein expression contributing to human lung fibroblast differentiation. In summary, active metabolite of vitamin D suppresses TGF- $\beta 1$-induced differentiation of human lung fibroblasts by miR-27b targets $3^{\prime}$-UTR of VDR; thus it may be a potential novel strategy of treatment in pathways of differentiation [91]. 


\section{Conclusion}

Animal models of asthma and isolated airway cell studies showed the role of miRNA in inflammation of airway and related asthma and COPD as well as remodeling and inflammation-related signaling pathways. Evidence presented in this review supports the idea that miRNAs are emerging as novelty therapeutic and diagnostic targets in inflammatory lung diseases.

\section{Competing Interests}

The authors declare that they have no competing interests.

\section{Acknowledgments}

This paper was funded by Medical University of Lodz (503/0149-03/503-01-002).

\section{References}

[1] R. Booton and M. A. Lindsay, "Emerging role of microRNAs and long noncoding RNAs in respiratory disease," Chest, vol. 146, no. 1, pp. 193-204, 2014.

[2] D. P. Bartel, "MicroRNAs: Genomics, Biogenesis, Mechanism, and Function," Cell, vol. 116, no. 2, pp. 281-297, 2004.

[3] R. C. Friedman, K. K.-H. Farh, C. B. Burge, and D. P. Bartel, "Most mammalian mRNAs are conserved targets of microRNAs," Genome Research, vol. 19, no. 1, pp. 92-105, 2009.

[4] M. M. Perry, I. M. Adcock, and K. F. Chung, "Role of microRNAs in allergic asthma: present and future," Current Opinion in Allergy and Clinical Immunology, vol. 15, no. 2, pp. 156-162, 2015.

[5] A. M. Denli, B. B. J. Tops, R. H. A. Plasterk, R. F. Ketting, and G. J. Hannon, "Processing of primary microRNAs by the Microprocessor complex," Nature, vol. 432, no. 7014, pp. 231235, 2004.

[6] R. I. Gregory, K.-P. Yan, G. Amuthan et al., "The Microprocessor complex mediates the genesis of microRNAs," Nature, vol. 432, no. 7014, pp. 235-240, 2004.

[7] J. Han, Y. Lee, K.-H. Yeom, Y.-K. Kim, H. Jin, and V. N. Kim, "The Drosha-DGCR8 complex in primary microRNA processing," Genes and Development, vol. 18, no. 24, pp. 30163027, 2004.

[8] B. M. Engels and G. Hutvagner, "Principles and effects of microRNA-mediated post-transcriptional gene regulation," Oncogene, vol. 25, no. 46, pp. 6163-6169, 2006.

[9] R. S. Pillai, S. N. Bhattacharyya, and W. Filipowicz, "Repression of protein synthesis by miRNAs: how many mechanisms?" Trends in Cell Biology, vol. 17, no. 3, pp. 118-126, 2007.

[10] C. A. Akdis, "Therapies for allergic inflammation: refining strategies to induce tolerance," Nature Medicine, vol. 18, no. 5, pp. 736-749, 2012.

[11] H. Renz, P. Brandtzaeg, and M. Hornef, "The impact of perinatal immune development on mucosal homeostasis and chronic inflammation," Nature Reviews Immunology, vol. 12, no. 1, pp. 9-23, 2012.

[12] R. Medzhitov, "Inflammation 2010: new adventures of an old flame," Cell, vol. 140, no. 6, pp. 771-776, 2010.

[13] M. P. Boldin and D. Baltimore, "MicroRNAs, new effectors and regulators of NF- $\kappa \mathrm{B}$," Immunological Reviews, vol. 246, no. 1, pp. 205-220, 2012.
[14] R. M. O'Connell, D. S. Rao, and D. Baltimore, "MicroRNA regulation of inflammatory responses," Annual Review of Immunology, vol. 30, pp. 295-312, 2012.

[15] L. A. O’Neill, F. J. Sheedy, and C. E. McCoy, "MicroRNAs: the fine-tuners of Toll-like receptor signalling," Nature Reviews Immunology, vol. 11, no. 3, pp. 163-175, 2011.

[16] S. E. Wenzel, "Asthma phenotypes: the evolution from clinical to molecular approaches," Nature Medicine, vol. 18, no. 5, pp. 716-725, 2012.

[17] A. Ebrahimi and E. Sadroddiny, "MicroRNAs in lung diseases: recent findings and their pathophysiological implications," Pulmonary Pharmacology and Therapeutics, vol. 34, pp. 55-63, 2015.

[18] T. X. Lu, A. Munitz, and M. E. Rothenberg, "MicroRNA-21 is up-regulated in allergic airway inflammation and regulates IL12 p35 expression," The Journal of Immunology, vol. 182, no. 8, pp. 4994-5002, 2009.

[19] T. X. Lu, J. Hartner, E.-J. Lim et al., "MicroRNA-21 limits in vivo immune response-mediated activation of the IL-12/IFN$\gamma$ pathway, Th1 polarization, and the severity of delayed-type hypersensitivity," Journal of Immunology, vol. 187, no. 6, pp. 3362-3373, 2011.

[20] M. Wang, Y. Huang, Z. Liang et al., "Plasma miRNAs might be promising biomarkers of chronic obstructive pulmonary disease," The Clinical Respiratory Journal, vol. 10, no. 1, pp. 104111, 2016.

[21] A. Sinha, A. K. Yadav, S. Chakraborty et al., "Exosome-enclosed microRNAs in exhaled breath hold potential for biomarker discovery in patients with pulmonary diseases," The Journal of Allergy and Clinical Immunology, vol. 132, no. 1, pp. 219-222.e7, 2013.

[22] A. N. Roff, T. J. Craig, A. August, C. Stellato, and F. T. Ishmael, "MicroRNA-570-3p regulates HuR and cytokine expression in airway epithelial cells," American Journal of Clinical and Experimental Immunology, vol. 3, no. 2, pp. 68-83, 2014.

[23] B. Levänen, N. R. Bhakta, P. Torregrosa Paredes et al., "Altered microRNA profiles in bronchoalveolar lavage fluid exosomes in asthmatic patients," Journal of Allergy and Clinical Immunology, vol. 131, no. 3, pp. 894-e8, 2013.

[24] M. Pinkerton, V. Chinchilli, E. Banta et al., "Differential expression of microRNAs in exhaled breath condensates of patients with asthma, patients with chronic obstructive pulmonary disease, and healthy adults," Journal of Allergy and Clinical Immunology, vol. 132, no. 1, pp. 217-219.e2, 2013.

[25] R. Trotta, L. Chen, S. Costinean et al., "Overexpression of miR155 causes expansion, arrest in terminal differentiation and functional activation of mouse natural killer cells," Blood, vol. 121, no. 16, pp. 3126-3134, 2013.

[26] M. M. Perry, J. E. Baker, D. S. Gibeon, I. M. Adcock, and K. F. Chung, "Airway smooth muscle hyperproliferation is regulated by MicroRNA-221 in severe asthma," American Journal of Respiratory Cell and Molecular Biology, vol. 50, no. 1, pp. 7-17, 2014.

[27] B. Bleck, G. Grunig, A. Chiu et al., "MicroRNA-375 regulation of thymic stromal lymphopoietin by diesel exhaust particles and ambient particulate matter in human bronchial epithelial cells," Journal of Immunology, vol. 190, no. 7, pp. 3757-3763, 2013.

[28] M. M. Perry, E. Tsitsiou, P. J. Austin et al., "Role of non-coding RNAs in maintaining primary airway smooth muscle cells," Respiratory Research, vol. 15, no. 1, article 58, 2014.

[29] D. Baumjohann and K. M. Ansel, "MicroRNA-mediated regulation of T helper cell differentiation and plasticity," Nature Reviews Immunology, vol. 13, no. 9, pp. 666-678, 2013. 
[30] S. Takyar, H. Vasavada, J.-G. Zhang et al., "VEGF controls lung Th2 inflammation via the miR-1-Mpl (myeloproliferative leukemia virus oncogene)-P-selectin axis," Journal of Experimental Medicine, vol. 210, no. 10, pp. 1993-2010, 2013.

[31] S. Molina-Pinelo, M. D. Pastor, R. Suarez et al., "MicroRNA clusters: dysregulation in lung adenocarcinoma and COPD," European Respiratory Journal, vol. 43, no. 6, pp. 1740-1749, 2014.

[32] X.-B. Wu, M.-Y. Wang, H.-Y. Zhu, S.-Q. Tang, Y.-D. You, and Y.-Q. Xie, "Overexpression of microRNA-21 and microRNA126 in the patients of bronchial asthma," International Journal of Clinical and Experimental Medicine, vol. 7, no. 5, pp. 1307-1312, 2014.

[33] B. S. Comer, B. Camoretti-Mercado, P. C. Kogut, A. J. Halayko, J. Solway, and W. T. Gerthoffer, "MicroRNA-146a and microRNA$146 \mathrm{~b}$ expression and anti-inflammatory function in human airway smooth muscle," American Journal of Physiology-Lung Cellular and Molecular Physiology, vol. 307, no. 9, pp. L727-L734, 2014.

[34] B. S. Comer, B. Camoretti-Mercado, P. C. Kogut, A. J. Halayko, J. Solway, and W. T. Gerthoffer, "Cyclooxygenase-2 and microrna155 expression are elevated in asthmatic airway smooth muscle cells," American Journal of Respiratory Cell and Molecular Biology, vol. 52, no. 4, pp. 438-447, 2015.

[35] R. Hu, W. Pan, A. V. Fedulov et al., "MicroRNA-10a controls airway smooth muscle cell proliferation via direct targeting of the PI3 kinase pathway," FASEB Journal, vol. 28, no. 5, pp. 23472357, 2014.

[36] R. C. Fry, J. E. Rager, R. Bauer et al., "Air toxics and epigenetic effects: ozone altered microRNAs in the sputum of human subjects," American Journal of Physiology-Lung Cellular and Molecular Physiology, vol. 306, no. 12, pp. L1129-L1137, 2014.

[37] J. N. Brown, H. M. Brewer, C. D. Nicora et al., "Protein and microRNA biomarkers from lavage, urine, and serum in military personnel evaluated for dyspnea," BMC Medical Genomics, vol. 7, no. 1, article 58, 2014.

[38] D. Naidoo, A. C. Wu, M. H. Brilliant et al., "A polymorphism in HLA-G modifies statin benefit in asthma," Pharmacogenomics Journal, vol. 15, no. 3, pp. 272-277, 2015.

[39] E. R. Sutherland and R. J. Martin, "Airway inflammation in chronic obstructive pulmonary disease: comparisons with asthma," Journal of Allergy and Clinical Immunology, vol. 112, no. 5, pp. 819-828, 2003.

[40] J. J. Soler-Cataluña, B. Cosío, J. L. Izquierdo et al., "Consensus document on the overlap phenotype COPD-asthma in COPD," Archivos de Bronconeumología (English Edition), vol. 48, no. 9, pp. 331-337, 2012.

[41] J. Ikari, L. M. Smith, A. J. Nelson et al., "Effect of culture conditions on microRNA expression in primary adult control and COPD lung fibroblasts in vitro," In Vitro Cellular and Developmental Biology-Animal, vol. 51, no. 4, pp. 390-399, 2015.

[42] H. Rupani, T. Sanchez-Elsner, and P. Howarth, "MicroRNAs and respiratory diseases," European Respiratory Journal, vol. 41, no. 3, pp. 695-705, 2013.

[43] Z. Cao, N. Zhang, T. Lou et al., "microRNA-183 down-regulates the expression of $\mathrm{BKCa} \beta 1$ protein that is related to the severity of chronic obstructive pulmonary disease," Hippokratia, vol. 18, no. 4, pp. 328-332, 2014.

[44] A. Donaldson, S. A. Natanek, A. Lewis et al., "Increased skeletal muscle-specific microRNA in the blood of patients with COPD," Thorax, vol. 68, no. 12, pp. 1140-1149, 2013.
[45] M. Schuliga, "NF-kappaB signaling in chronic inflammatory airway disease," Biomolecules, vol. 5, no. 3, pp. 1266-1283, 2015.

[46] X. Jiang, L. Du, L. Wang et al., "Serum microRNA expression signatures identified from genome-wide microRNA profiling serve as novel noninvasive biomarkers for diagnosis and recurrence of bladder cancer," International Journal of Cancer, vol. 136, no. 4, pp. 854-862, 2015.

[47] E. Puig-Vilanova, R. Aguiló, A. Rodríguez-Fuster, J. MartínezLlorens, J. Gea, and E. Barreiro, "Epigenetic mechanisms in respiratory muscle dysfunction of patients with chronic obstructive pulmonary disease," PloS ONE, vol. 9, no. 11, Article ID e111514, 2014

[48] M. J. Toth, M. S. Miller, D. M. Callahan et al., "Molecular mechanisms underlying skeletal muscle weakness in human cancer: reduced myosin-actin cross-bridge formation and kinetics," Journal of Applied Physiology, vol. 114, no. 7, pp. 858-868, 2013.

[49] S. Mizuno, H. J. Bogaard, J. Gomez-Arroyo et al., "MicroRNA$199 a-5 p$ is associated with hypoxia-inducible factor- $1 \alpha$ expression in lungs from patients with COPD," Chest, vol. 142, no. 3, pp. 663-672, 2012.

[50] T. Hassan, S. G. J. Smith, K. Gaughan et al., "Isolation and identification of cell-specific microRNAs targeting a messenger RNA using a biotinylated anti-sense oligonucleotide capture affinity technique," Nucleic Acids Research, vol. 41, no. 6, article e71, 2013.

[51] S. M. Janciauskiene, R. Bals, R. Koczulla, C. Vogelmeier, T. Köhnlein, and T. Welte, "The discovery of $\alpha 1$-antitrypsin and its role in health and disease," Respiratory Medicine, vol. 105, no. 8, pp. 1129-1139, 2011.

[52] M. Zago, A. Rico de Souza, E. Hecht et al., "The NF- $\kappa$ B family member RelB regulates microRNA miR-146a to suppress cigarette smoke-induced COX-2 protein expression in lung fibroblasts," Toxicology Letters, vol. 226, no. 2, pp. 107-116, 2014.

[53] I. Ogulur, G. Gurhan, F. E. Kombak, D. Filinte, I. Barlan, and T. Akkoc, "Allogeneic pluripotent stem cells suppress airway inflammation in murine model of acute asthma," International Immunopharmacology, vol. 22, no. 1, pp. 31-40, 2014.

[54] T. W. Guilbert, W. J. Morgan, R. S. Zeiger et al., "Long-term inhaled corticosteroids in preschool children at high risk for asthma," The New England Journal of Medicine, vol. 354, no. 19, pp. 1985-1997, 2006.

[55] J. L. J. van der Velden, S. M. Hoffman, J. F. Alcorn et al., "Absence of c-Jun NH2-terminal kinase 1 protects against house dust mite-induced pulmonary remodeling but not airway hyperresponsiveness and inflammation," American Journal of Physiology_Lung Cellular and Molecular Physiology, vol. 306, no. 9, pp. L866-L875, 2014.

[56] I. Haj-Salem, R. Fakhfakh, J.-C. Bérubé et al., "MicroRNA-19a enhances proliferation of bronchial epithelial cells by targeting TGF $\beta$ R2 gene in severe asthma," Allergy, vol. 70, no. 2, pp. 212219, 2015.

[57] T. Makinde, R. F. Murphy, and D. K. Agrawal, “The regulatory role of TGF- $\beta$ in airway remodeling in asthma," Immunology and Cell Biology, vol. 85, no. 5, pp. 348-356, 2007.

[58] S. D. Brown, K. M. Baxter, S. T. Stephenson, A. M. Esper, L. A. S. Brown, and A. M. Fitzpatrick, "Airway TGF- $\beta 1$ and oxidant stress in children with severe asthma: association with airflow limitation," The Journal of Allergy and Clinical Immunology, vol. 129, no. 2, pp. 388-396.e8, 2012.

[59] G. Chen and N. Khalil, "TGF- $\beta 1$ increases proliferation of airway smooth muscle cells by phosphorylation of map kinases," Respiratory Research, vol. 7, article 2, 2006. 
[60] J. Ma, Q. Wang, T. Fei, J.-D. J. Han, and Y.-G. Chen, "MCP-1 mediates TGF- $\beta$-induced angiogenesis by stimulating vascular smooth muscle cell migration," Blood, vol. 109, no. 3, pp. 987994, 2007.

[61] D. Ierodiakonou, D. S. Postma, G. H. Koppelman et al., "TGF$\beta 1$ polymorphisms and asthma severity, airway inflammation, and remodeling," Journal of Allergy and Clinical Immunology, vol. 131, no. 2, pp. 582-585, 2013.

[62] A. L. Degryse, H. Tanjore, X. C. Xu et al., “TGF $\beta$ signaling in lung epithelium regulates bleomycin-induced alveolar injury and fibroblast recruitment," American Journal of PhysiologyLung Cellular and Molecular Physiology, vol. 300, no. 6, pp. L887-L897, 2011.

[63] M. Chen, L. Huang, W. Zhang et al., "MiR-23b controls TGF- $\beta 1$ induced airway smooth muscle cell proliferation via TGF $\beta$ R2/p-Smad3 signals," Molecular Immunology, vol. 70, pp. 84-93, 2016.

[64] C. Malmhäll, S. Alawieh, Y. Lu et al., "MicroRNA-155 is essential for TH2-mediated allergen-induced eosinophilic inflammation in the lung," Journal of Allergy and Clinical Immunology, vol. 133, no. 5, pp. 1438.e1-1438.e7, 2014.

[65] H. Hu, J. M. Bliss, Y. Wang, and J. Colicelli, "RIN1 is an ABL tyrosine kinase activator and a regulator of epithelial-cell adhesion and migration," Current Biology, vol. 15, no. 9, pp. 815823, 2005.

[66] C. Ring, M. H. Ginsberg, J. Haling, and A. M. Pendergast, "Ablinteractor-1 (Abil) has a role in cardiovascular and placental development and is a binding partner of the $\alpha 4$ integrin," Proceedings of the National Academy of Sciences of the United States of America, vol. 108, no. 1, pp. 149-154, 2011.

[67] R. A. Cleary, R. Wang, O. Waqar, H. A. Singer, and D. D. Tang, "Role of c-Abl tyrosine kinase in smooth muscle cell migration," American Journal of Physiology - Cell Physiology, vol. 306, no. 8, pp. C753-C761, 2014.

[68] C. Widmann, S. Gibson, M. B. Jarpe, and G. L. Johnson, "Mitogen-activated protein kinase: conservation of a threekinase module from yeast to human," Physiological Reviews, vol. 79, no. 1, pp. 143-180, 1999.

[69] K. Vallières, É. Petitclerc, and G. Laroche, "On the ability of imatinib mesylate to inhibit smooth muscle cell proliferation without delaying endothelialization: an in vitro study," Vascular Pharmacology, vol. 51, no. 1, pp. 50-56, 2009.

[70] L. Jia, R. Wang, and D. D. Tang, "Abl regulates smooth muscle cell proliferation by modulating actin dynamics and ERK1/2 activation," American Journal of Physiology-Cell Physiology, vol. 302, no. 7, pp. C1026-C1034, 2012.

[71] R. Wang, O. P. Mercaitis, L. Jia, R. A. Panettieri, and D. D. Tang, "Raf-1, actin dynamics, and abelson tyrosine kinase in human airway smooth muscle cells," American Journal of Respiratory Cell and Molecular Biology, vol. 48, no. 2, pp. 172-178, 2013.

[72] R. A. Cleary, R. Wang, T. Wang, and D. D. Tang, "Role of $\mathrm{Abl}$ in airway hyperresponsiveness and airway remodeling," Respiratory Research, vol. 14, article 105, 2013.

[73] R. Wang, R. A. Cleary, T. Wang, J. Li, and D. D. Tang, "The association of cortactin with profilin-1 is critical for smooth muscle contraction," The Journal of Biological Chemistry, vol. 289, no. 20, pp. 14157-14169, 2014.

[74] C. K. Rhee, J. W. Kim, C. K. Park et al., "Effect of imatinib on airway smooth muscle thickening in a murine model of chronic asthma," International Archives of Allergy and Immunology, vol. 155, no. 3, pp. 243-251, 2011.
[75] G. Liao, R. A. Panettieri, and D. D. Tang, "MicroRNA-203 negatively regulates c-Abl, ERK1/2 phosphorylation, and proliferation in smooth muscle cells," Physiological Reports, vol. 3, no. 9, Article ID e12541, 2015.

[76] L. Shi, Q. Xin, R. Chai, L. Liu, and Z. Ma, "Ectopic expressed miR-203 contributes to chronic obstructive pulmonary disease via targeting TAK1 and PIK3CA," International Journal of Clinical and Experimental Pathology, vol. 8, no. 9, pp. 1066210670, 2015.

[77] Y. Liu, K. Yang, H. Shi et al., "MiR-21 modulates human airway smooth muscle cell proliferation and migration in asthma through regulation of PTEN expression," Experimental Lung Research, vol. 41, no. 10, pp. 535-545, 2015.

[78] E. Alexandrova, N. Miglino, A. Hashim et al., "Small RNA profiling reveals deregulated phosphatase and tensin homolog (PTEN)/phosphoinositide 3-kinase (PI3K)/Akt pathway in bronchial smooth muscle cells from asthmatic patients," Journal of Allergy and Clinical Immunology, vol. 137, no. 1, pp. 58-67, 2016.

[79] S. T. Murphy, G. Alton, S. Bailey et al., "Discovery of novel, potent, and selective inhibitors of 3-phosphoinositidedependent kinase (PDK1)," Journal of Medicinal Chemistry, vol. 54, no. 24, pp. 8490-8500, 2011.

[80] Y. Liu, K. Yang, X. Sun et al., "MiR-138 suppresses airway smooth muscle cell proliferation through the PI3K/AKT signaling pathway by targeting PDK1," Experimental Lung Research, vol. 41, no. 7, pp. 363-369, 2015.

[81] K. R. Stenmark, M. G. Frid, and M. E. Yeager, "Fibrocytes: potential new therapeutic targets for pulmonary hypertension?" European Respiratory Journal, vol. 36, no. 6, pp. 1232-1235, 2010.

[82] S. Sartore, A. Chiavegato, E. Faggin et al., "Contribution of adventitial fibroblasts to neointima formation and vascular remodeling: from innocent bystander to active participant," Circulation Research, vol. 89, no. 12, pp. 1111-1121, 2001.

[83] X. Liu, A. Nelson, X. Wang et al., "Vitamin D modulates prostaglandin E2 synthesis and degradation in human lung fibroblasts," American Journal of Respiratory Cell and Molecular Biology, vol. 50, no. 1, pp. 40-50, 2014.

[84] M. Hetzel, M. Bachem, D. Anders, G. Trischler, and M. Faehling, "Different effects of growth factors on proliferation and matrix production of normal and fibrotic human lung fibroblasts," Lung, vol. 183, no. 4, pp. 225-237, 2005.

[85] M. Coen, G. Gabbiani, and M.-L. Bochaton-Piallat, "Myofibroblast-mediated adventitial remodeling: an underestimated player in arterial pathology," Arteriosclerosis, Thrombosis, and Vascular Biology, vol. 31, no. 11, pp. 2391-2396, 2011.

[86] J. S. Duffield, M. Lupher, V. J. Thannickal, and T. A. Wynn, "Host responses in tissue repair and fibrosis," Annual Review of Pathology: Mechanisms of Disease, vol. 8, pp. 241-276, 2013.

[87] A. Biernacka, M. Dobaczewski, and N. G. Frangogiannis, "TGF$\beta$ signaling in fibrosis," Growth Factors, vol. 29, no. 5, pp. 196202, 2011.

[88] P. A. Suwanabol, S. M. Seedial, F. Zhang et al., “TGF- $\beta$ and Smad3 modulate PI3K/Akt signaling pathway in vascular smooth muscle cells," American Journal of Physiology-Heart and Circulatory Physiology, vol. 302, no. 11, pp. H2211-H2219, 2012.

[89] L. Ma and W. K. Chung, "The genetic basis of pulmonary arterial hypertension," Human Genetics, vol. 133, no. 5, pp. 471479, 2014.

[90] D. Kajdaniuk, B. Marek, H. Borgiel-Marek, and B. Kos-Kudła, "Transforming growth factor $\beta 1$ (TGF $\beta 1$ ) in physiology and 
pathology," Endokrynologia Polska, vol. 64, no. 5, pp. 384-396, 2013.

[91] F. Li, A. Zhang, Y. Shi, Y. Ma, and Y. Du, " $1 \alpha, 25-D i h y d r o x-$ yvitamin D3 prevents the differentiation of human lung fibroblasts via microRNA-27b targeting the Vitamin D receptor," International Journal of Molecular Medicine, vol. 36, no. 4, pp. 967-974, 2015. 


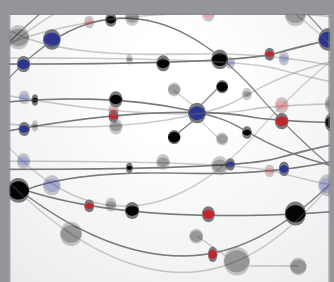

The Scientific World Journal
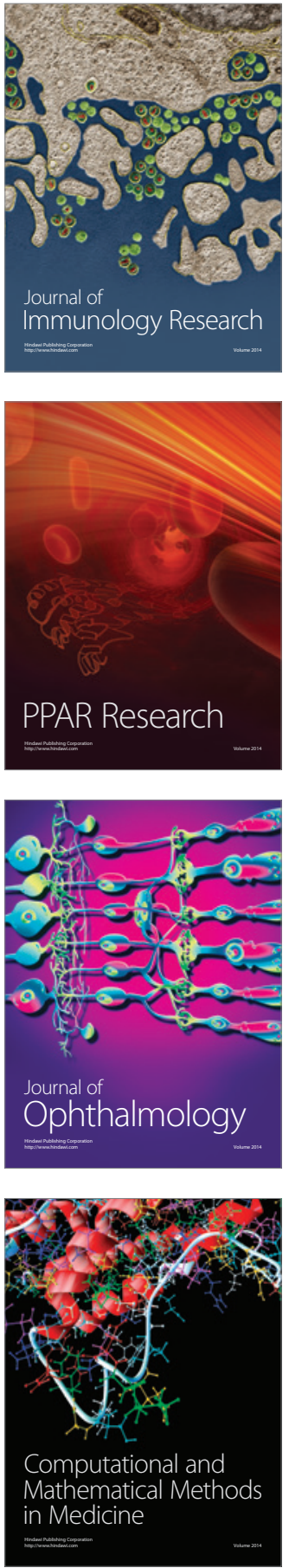

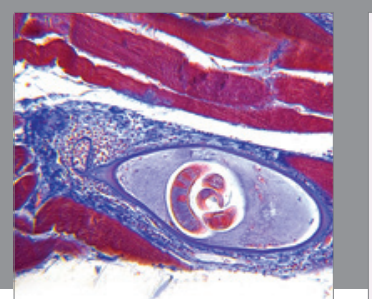

Gastroenterology Research and Practice

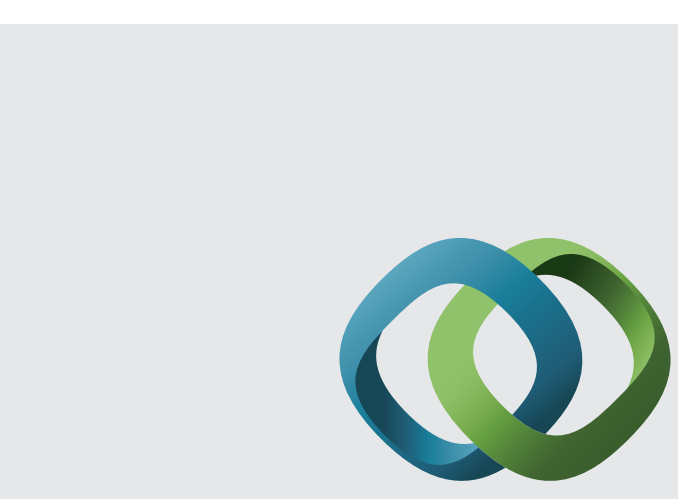

\section{Hindawi}

Submit your manuscripts at

http://www.hindawi.com
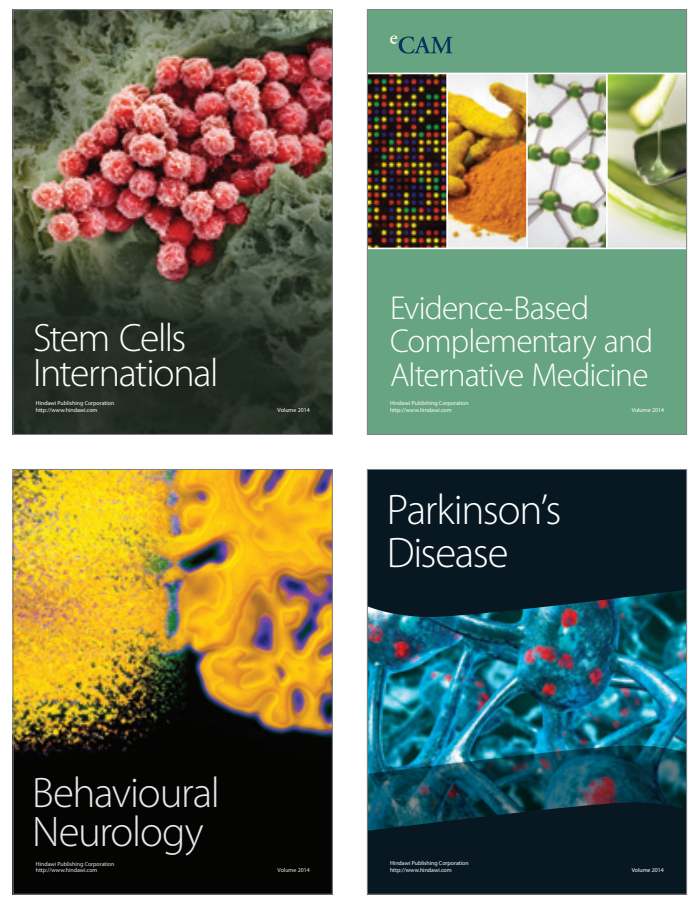
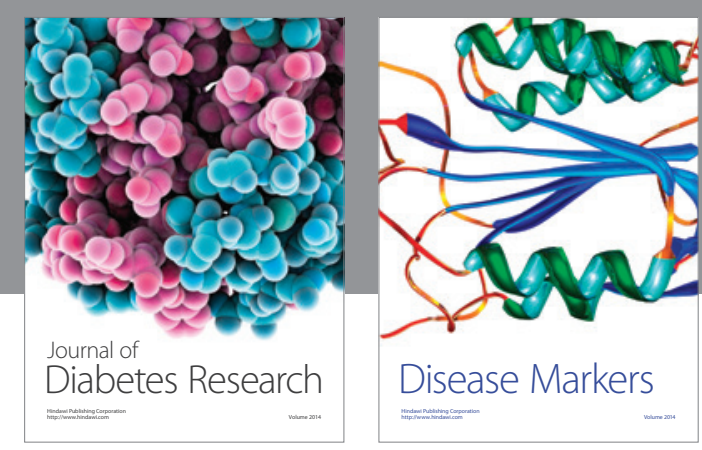

Disease Markers
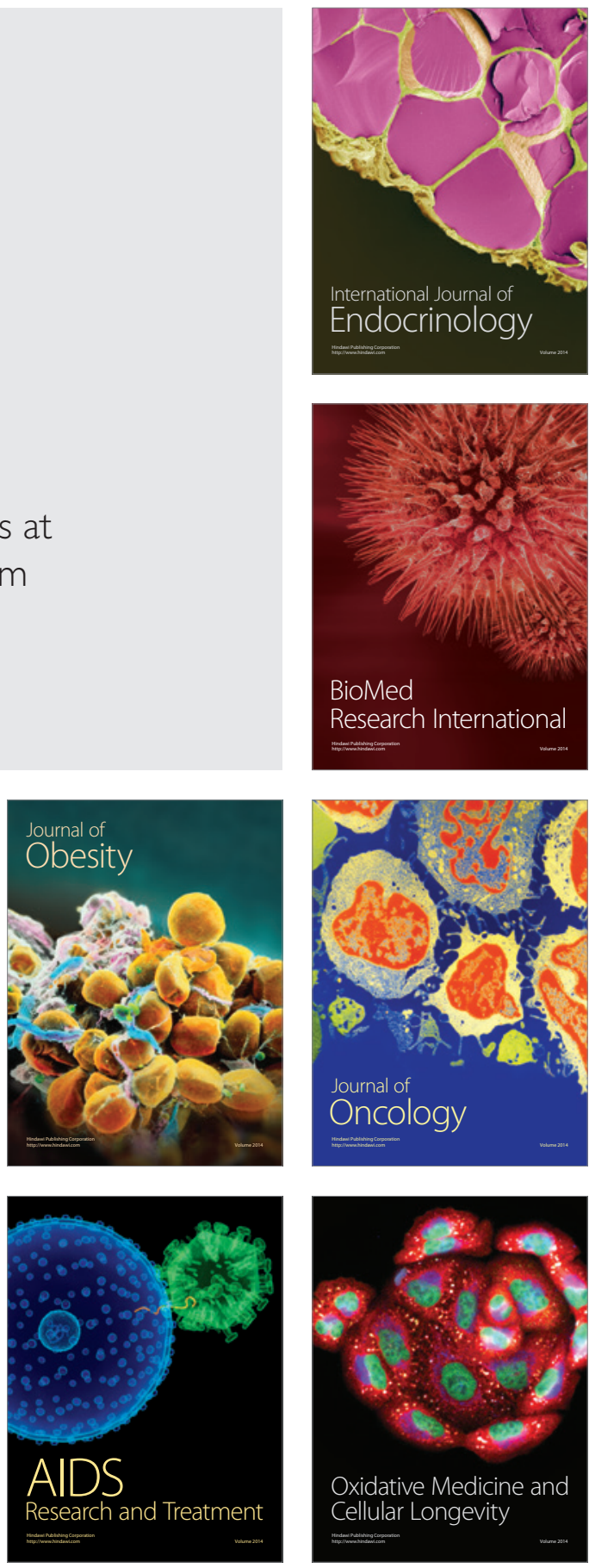